\title{
A systematic review and meta-analysis of interventions for the treatment of chronic non- hypovolaemic hypotonic hyponatraemia
}

\author{
McCool R ${ }^{1}$, Glanville J11, Quinn J2, O'Reilly K², Petrakova L², Jamookeeah C2 \\ ${ }^{1}$ York Health Economics Consortium, York, UK. ${ }^{2}$ Otsuka Pharmaceutical Europe Ltd., Wexham, UK.
}

\section{BACKGROUND AND OBJECTIVES}

International and national guidelines on the treatment of chronic nonhypovolaemic hypotonic hyponatraemia differ widely in their recommendations. We used Cochrane methods to conduct a systematic review and meta-analysis (PROSPERO registration number: CRD42015016670) of the effectiveness and safety of interventions for this condition.

\section{Figure 1: $\quad$ Study selection (PRISMA Diagram)}

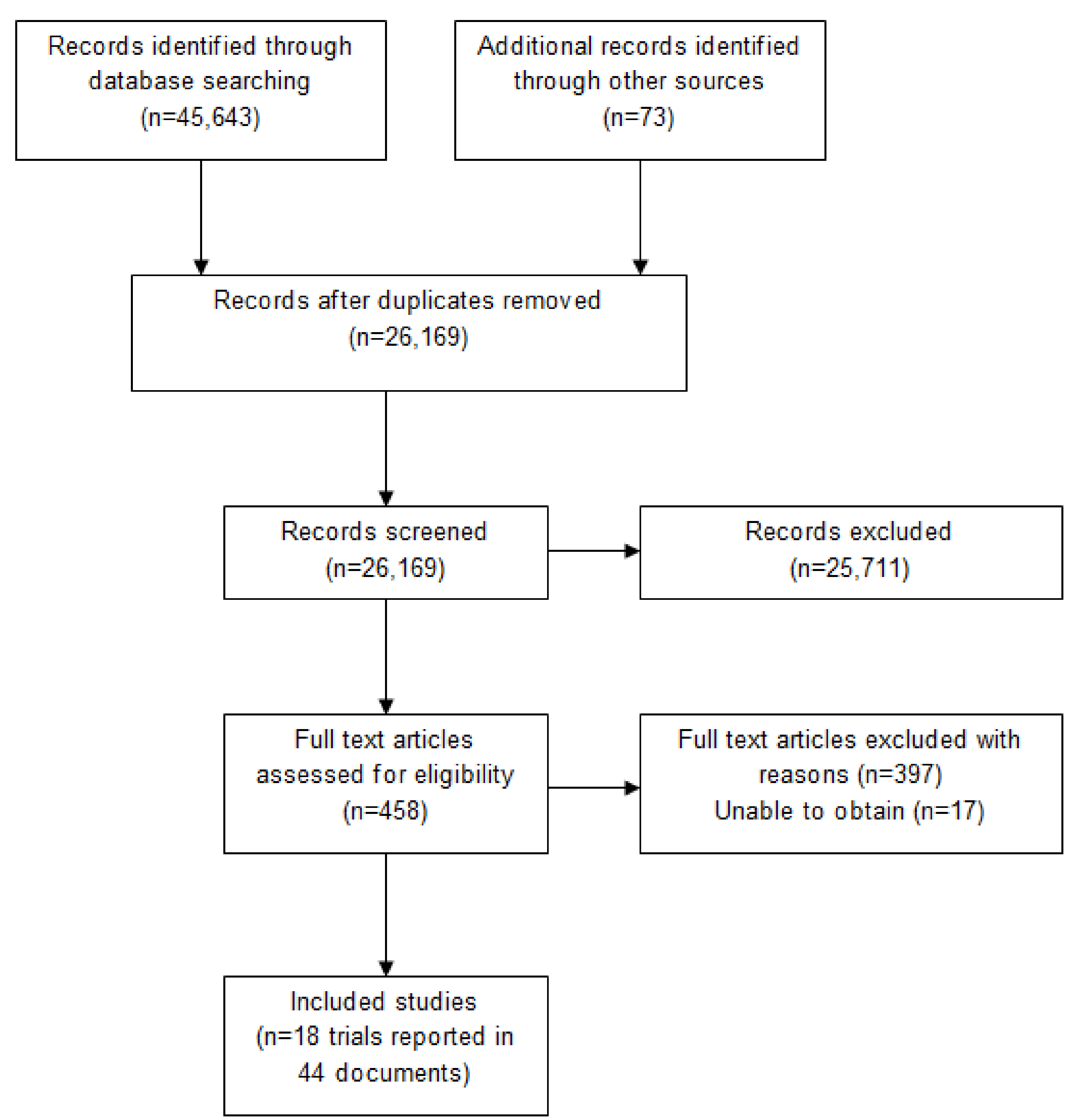

\section{METHODS}

Eligible studies were randomised or quasi-randomised controlled trials published in English that compared any degree of fluid restriction or any drug treatment with the aim of increasing serum sodium concentration in patients with chronic non-hypovolaemic hypotonic hyponatraemia. A range of outcomes were specified including: change in serum sodium concentration $(\mathrm{mmol} / \mathrm{L}$ ), response (defined as an increase in serum sodium of $\geq 5 \mathrm{mmol} / \mathrm{L}$ or normalisation of serum sodium concentration ( $\geq 135$ to $145 \mathrm{mmol} / \mathrm{L}$ ), mortality and discontinuation, which are reported here. Outcomes relating to health related quality of life, length of hospital stay, outcomes related to overcorrection of serum sodium concentration and adverse events were also investigated but are not reported here. The review protocol was registered with PROSPERO.

Systematic literature searches were conducted in 7 databases (including Medline and Embase) as well as the websites of relevant conference proceedings up to February 2015

Studies were screened by two independent reviewers against the eligibility criteria. Any disagreements were discussed with a third reviewer. Data extraction and quality assessment was carried out by two independent reviewers. Key data were collected on the trial design and methodology, patient population, details of interventions and relevant outcomes. Risk of bias was assessed using the Cochrane Risk of Bias tool (1). Where appropriate, outcome data were combined using both fixed- and random-effects models in a meta-analysis using RevMan (version 5.3) (2). Results of the random effects model were deemed most appropriate and are reported here. A sensitivity analysis was conducted excluding studies with a high risk of bias.

\section{RESULTS}

The searches retrieved 45,716 records and 18 trials met the eligibility criteria or reported data for an eligible subgroup of patients; 2 trials assessed conivaptan, 4 trials assessed lixivaptan, 3 trials assessed satavaptan, and 9 trials assessed tolvaptan*. No RCT evidence was identified in relation to the alternative strategies commonly employed such as oral urea, salt tablets, mannitol, loop diuretics, demeclocycline and lithium.

Following an assessment of the risk of bias of the included trials, 5 trials were judged to be of a high risk, 12 were deemed to have an unclear risk and only one trial had a low risk of bias.

In both the individual trials and in the pooled analyses, all four "vaptans" were found to significantly improve serum sodium concentration and were associated with greater rates of response compared to placebo, with the exception of tolvaptan at day 14. There was no evidence of a difference between any of the vaptans compared with placebo for mortality, discontinuation and rates of over-correction in the individual trials or pooled analyses.

The sensitivity analyses excluding studies with a high risk of bias did not significantly alter the results of the meta-analyses.

\begin{tabular}{|c|c|c|c|c|c|}
\hline Table 1: & Resuli & ts of the & eta-analy & & \\
\hline \multirow{2}{*}{ Intervention } & \multirow{2}{*}{$\begin{array}{l}\text { Time point } \\
\text { (days) }\end{array}$} & \multicolumn{2}{|c|}{$\begin{array}{l}\text { Change in serum sodium } \\
\text { concentration }\end{array}$} & \multicolumn{2}{|c|}{ Response } \\
\hline & & $\begin{array}{l}\text { Number of } \\
\text { studies }\end{array}$ & $\begin{array}{c}\text { Mean Difference } \\
{[95 \% \mathrm{Cl}]}\end{array}$ & $\begin{array}{l}\text { Number of } \\
\text { studies }\end{array}$ & $\begin{array}{l}\text { Relative Risk } \\
\text { [95\% } \mathrm{Cl}]\end{array}$ \\
\hline Conivaptan & 5 & 2 & $5.34[2.82,7.86]$ & - & - \\
\hline \multirow{2}{*}{ Lixivaptan } & 7 & 4 & $2.82[1.70,3.95]$ & 3 & $2.81[1.34,5.91]$ \\
\hline & 56 & - & - & 1 & $1.39[1.03,1.88]$ \\
\hline \multirow{4}{*}{ Tolvaptan } & 4 & 5 & $3.98[2.31,5.65]$ & 4 & $3.30[1.97,5.54]$ \\
\hline & 7 & - & - & 4 & $2.22[1.25,3.93]$ \\
\hline & 14 & 4 & $4.22[2.09,6.34]$ & 2 & $4.24[0.55,32.40]$ \\
\hline & $25-30$ & 3 & $3.54[2.15,4.94]$ & 3 & $2.25[1.74,2.89]$ \\
\hline Satavaptan & $4-5$ & 3 & $4.95[2.81,7.10]$ & 3 & $2.57[1.66,4.00]$ \\
\hline
\end{tabular}

\section{CONCLUSIONS}

The RCT evidence indicates that each of the vaptans significantly improves serum sodium concentration compared with placebo and is associated with greater rates of response than placebo. Few studies measured health-related quality of life (QoL) using a range of different instruments and showed no evidence that vaptans negatively impact QoL. For all vaptans, cases of overcorrection were rare. There was no evidence of a difference between any of the vaptans compared with placebo for mortality, discontinuation or rates of hypernatremia. With the exception of vaptans, no RCT evidence was identified in relation to the alternative strategies commonly employed including urea, mannitol, loop diuretics, corticosteroids, demeclocycline, lithium and phenytoin. Further head to head RCTs are required to determine the comparative efficacy of other treatments currently used in clinical practice or recommended in guidelines (hypertonic saline, oral urea, salt tablets, loop diuretics, mannitol, demeclocycline, lithium) for chronic hypotonic nonhypovolaemic hyponatraemia.

REFERENCES

1. Higgins JP, Green S. Cochrane handbook for systematic reviews of interventions: The Cochrane Collaboration, 2011. 2. Review Manager (RevMan) [Computer program]. Version 5.3. Copenhagen: The Nordic Cochrane Centre, The Cochrane Collaboration, 2014.
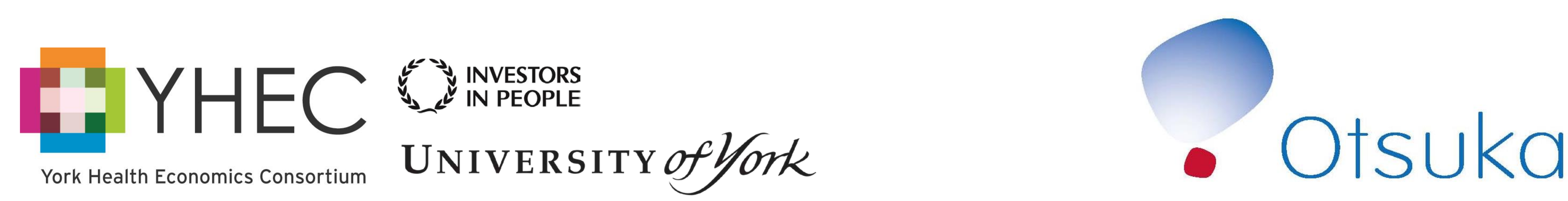